\title{
Complicaciones del Manejo Quirúrgico De Los Pacientes Con Trauma Penetrante De Tórax En Un Hospital Universitario Al Sur De Colombia
}

\author{
${ }^{1}$ Ana M Cuellar, ${ }^{2}$ José D Charry, ${ }^{3}$ Rolando Medina, ${ }^{4}$ Oliver Chávarro
}

\section{RESUMEN}

Introducción y Objetivos: El trauma es considerado un problema de salud pública. Es una de las patologías que generan mayor discapacidad y mortalidad a nivel de Latinoamérica y Colombia no es la excepción. El objetivo de este estudio fue describir las principales complicaciones del manejo quirúrgico de los pacientes con trauma penetrante de tórax en un hospital universitario en el sur de Colombia.

Materiales y métodos: Estudio descriptivo observacional de los pacientes victimas de trauma penetrante de tórax que ingresaron entre enero de 2013 a marzo de 2015; Se evaluaron las variables clínicas, sociodemográficas, complicaciones, factores de riesgo y severidad de las lesiones; se realizó un análisis univariado, se calcularon medidas de tendencia central y de dispersión para las variables continuas, se utilizó la prueba de $\mathrm{Chi}^{2}$ para medir significancia estadística en el software en línea $R$ versión 2.15.2.

Resultados: En total se analizaron 485 pacientes, 8,65\% (42) desarrollaron alguna complicación; entre los pacientes en los que se registró algún tipo de complicación relacionada con el manejo quirúrgico del trauma penetrante de tórax, el empiema fue la principal complicación $59,52 \%$ (25), seguido por neumonía $28,57 \%$ (12), y SDRA $14,91 \%$ (5). La media de la edad fue de 34,2 $\pm 8,23$ años, la media del ISS fue de 16,4 $\pm 2,91$, el $88,1 \%$ de las lesiones fue por arma corto punzante, en $61,9 \%$ de los casos se presentó hemotórax, 30,95\% neumotórax y en $7,14 \%$ se presentó neumo - hemotorax. La media de la estancia hospitalaria fue de 14,8 $\pm 2,45$ días.

Conclusiones: Las complicaciones postquirúrgicas del manejo en el trauma penetrante de tórax se presentan en un hospital universitario al sur de Colombia. El empiema postraumático es la principal complicación que se presenta en los pacientes con trauma penetrante de tórax, uno de los factores de riesgo más importante es el hemotorax coagulado el cual podría identificarse y tratarse a tiempo; evitando comorbilidades durante la estancia hospitalaria.

\footnotetext{
${ }^{1,2}$ Médico interna, ${ }^{3,4}$ Cirujano General

1,2Médico Interna del servicio de Cirugía General, Hospital Universitario de Neiva, Universidad Surcolombiana, Neiva Huila, Colombia

${ }^{3,4}$ Departamento de Cirugía General Hospital Universitario de Neiva, Universidad Surcolombiana, Neiva, Huila, Colombia

Corresponding Author: Ana M Cuellar, Médico interna, Médico Interna del servicio de Cirugía General, Hospital Universitario de Neiva, Universidad Surcolombiana, Neiva Huila Colombia, teléfono: +573158944410, e-mail: anamariacuellarb@ gmail.com
}

Palabras clave: Complicaciones postquirúrgicas, Tórax, Trauma penetrante.

\section{ABSTRACT}

Introduction and Objectives: Trauma is considered a public health problem. It is one of the pathologies which leads to greater level of disability and mortality in Latin America, and Colombia is not an exception. The aim of this study was to describe the main complications of surgical management of patients with penetrating thoracic trauma in a university hospital in southern Colombia.

Materials and methods: A descriptive study was conducted on patients who were the victims of penetrating chest trauma, admitted from January 2013 to March 2015. Clinical, sociodemographic, complications, risk factors, and severity of lesions were evaluated; univariate analysis was performed; measures of central tendency and dispersion for continuous variables were calculated; Chi-square test was used to measure statistical significance in the online software $\mathrm{R}$ version 2.15.2.

Results: A total of 485 patients were analyzed, $8.65 \%$ (42) developed some complications. Among patients with some type of complication related to the surgical management of penetrating chest trauma, empyema was the main complication $59.52 \%$ (25), followed by pneumonia $28.57 \%$ (12) and ARDS $14,91 \%(5)$. The mean age was $34.2 \pm 8.23$ years, the mean ISS was $16.4 \pm 2.91$. Around $88.1 \%$ of the injuries were stabbing wounds, $61.9 \%$ cases presented hemothorax, $30.95 \%$ pneumothorax and $7,14 \%$ hemopneumothorax respectively. The average hospital stay was $14.8 \pm 2.45$ days.

Conclusion: Surgical complications after the operation in penetrating chest trauma are presented in a university hospital in southern Colombia. Posttraumatic empyema is the main complication that occurs in patients with penetrating chest trauma. One of the most important risk factor is coagulated hemothorax which could be identified and treated in time, avoiding comorbidities during the hospital stay.

Keywords: Penetrating trauma, Postsurgical complications, Thorax.

How to cite this article: Cuellar AM, Charry JD, Medina R, Chávarro O. Complicaciones del Manejo Quirúrgico De Los Pacientes Con Trauma Penetrante De Tórax En Un Hospital Universitario AI Sur De Colombia. Panam J Trauma Crit Care Emerg Surg 2017;6(3):142-145.

Source of support: Nil

Conflict of interest: None 


\section{INTRODUCCIÓN}

El trauma es considerado un problema de salud pública en el mundo, debido a la morbimortalidad que se desencadena secundario a éste tanto en países desarrollados como en vía de desarrollo. ${ }^{1}$ Teniendo en cuenta lo anterior, se ha reportado que el trauma de tórax es una de las mayores causas de hospitalización en el mundo y corresponde al 10-15\% de los traumas, ${ }^{2}$ con una mortalidad secundaria al trauma del $20-25 \%{ }^{3}$ a pesar de que hay estadísticas de otros países en los cuales se reportan tasas de mortalidad menores con un rango entre $4-20 \%{ }^{4}$

De los traumas de tórax, el penetrante corresponde al $40 \%{ }^{5}$ de los pacientes, aunque hay otros estudios que reportan tasas menores correspondiendo al $34 \%{ }^{2}$ y en algunos otros, valores tan bajos como $0.8 \%{ }^{4}$ La variación entre las frecuencias de presentación del trauma penetrante son atribuidos a ambientes violentos. ${ }^{3}$

El trauma penetrante se genera secundario a la aplicación directa y abrupta de una fuerza mecánica en un área focal. ${ }^{6}$ Existen tres mecanismos principales los cuales son causales de éste tipo de trauma y son principalmente por proyectil de arma de fuego, por arma cortopunzante o por empalamiento. ${ }^{7}$ Según el mecanismo traumático variará el grado de lesión provocada; puesto que, en el caso de las heridas por proyectil de arma de fuego se deben considerar las características propias del proyectil, la biomecánica y sobre todo la velocidad con la que impactará el tejido debido a que habrá mayor intercambio de energía entre el tejido y el elemento lesivo. En el caso del segundo mecanismo las lesiones dependerán del trayecto por el cual ingrese el arma para de esta forma establecer las estructuras potencialmente afectadas ${ }^{8}$ finalmente el empalamiento se presenta con mucha menor frecuencia y se produce por penetración de objetos elongados a un órgano. ${ }^{9}$

Para el manejo de este tipo de patología traumática va desde las medidas generales de soporte hemodinámico 9 y el manejo quirúrgico. ${ }^{10}$ Considerando lo anterior se ha descrito que las complicaciones secundarias al manejo de la patología traumática contribuyen a estancia hospitalaria prolongada y muerte tardía. ${ }^{5}$ Algunas de las que se han descrito son el empiema, fuga de aire persistente, neumonía, sangrado persistente, requerimiento de ventilación mecánica en el caso de los pacientes que necesitaron intervención pulmonar ${ }^{11}$ y en el caso de los pacientes que fueron manejados quirúrgicamente sólo mediante toracostomía cerrada; las complicaciones están relacionadas con la lesión de órganos intraabdominales e intratorácicos (esófago, estómago, intestino hígado, bazo, diafragma, sistema cardiovascular) y otras como fístulas broncopleurales, neumotórax recurrente, sangrado intercostal, fibrotórax, quilotórax, empiema entre otras. ${ }^{12}$
Teniendo en cuenta el gran impacto que tiene el trauma de tórax dentro del grupo de patologías traumáticas y las repercusiones que tienen las complicaciones del manejo quirúrgico de los pacientes en el tiempo de estancia hospitalaria y en la morbimortalidad se decide realizar este estudio cuyo principal objetivo es describir cuáles son las complicaciones que se presentan con mayor frecuencia en estos pacientes.

\section{MATERIALES Y MÉTODOS}

El presente es un estudio descriptivo observacional, transversal y retrospectivo el cual se realizó en el área de cirugía general de un hospital de tercer nivel en trauma.

Se recolectaron datos de las historias clínicas de pacientes que ingresaron con diagnóstico de trauma penetrante de tórax en un periodo comprendido entre enero del 2013 y marzo del 2015.

Se realizó muestreo no probabilístico y se incluyeron en el estudio aquellos pacientes de ambos sexos, mayores de 18 años de edad, hospitalizados por parte del servicio de cirugía general y que hubieran ingresado con diagnóstico de trauma penetrante de tórax. Se excluyeron aquellos que estuvieran en estado de embarazo, tuvieran diagnóstico de muerte cerebral, y quienes fallecieron antes de realizarse el procedimiento quirúrgico, al igual que los menores de 18 años. Se consignaron variables sociodemográficas, índice de shock, puntaje de severidad de la injuria, procedimientos quirúrgicos realizados, tipo de lesión torácica secundaria al trauma, requerimiento de ventilación mecánica invasiva, tiempo de estancia en unidad de cuidado intensivo, el tipo de complicación que presentó el paciente y mortalidad.

El diagnóstico de las complicaciones se realizó mediante los criterios que se comentarán a continuación: neumonía definida como la presencia de leucocitosis $>12000$, temperatura $>38.5^{\circ} \mathrm{C}$, evidencia radiográfica de consolidaciones o infiltrados y cultivo de esputo positivo. El empiema se diagnosticó cuando había leucocitosis $>12000$, temperatura $>38.5^{\circ} \mathrm{c}, \mathrm{LDH}>500$ UI y cultivos pleurales o gram de cultivo pleural positivo y el SDRA (síndrome de distrés respiratorio agudo) como desarrollo agudo de falla respiratoria, infiltrados bilaterales en radiografía de tórax, hipoxemia $\left(\mathrm{PaO}_{2} / \mathrm{FIO}_{2}\right)$ menor o igual a $200 \mathrm{~mm} \mathrm{Hg}$.

Los datos obtenidos en el estudio fueron almacenados en una interfaz del software estadístico en línea $R$ versión 2.15.2, el cual es un lenguaje y entorno de programación para análisis estadístico y gráfico, que proporciona herramientas estadísticas (modelos lineales y no lineales, test estadísticos, análisis de series temporales, algoritmos de clasificación y agrupamiento, etc.). Se calcularon medidas de tendencia central y dispersión para las variables 
Tabla 1: Hallazgos clínicas de los pacientes

\begin{tabular}{lll}
\hline & $n=485$ & $P<0.05$ \\
\hline Neumotórax & $150(30.95 \%)$ & 0.247 \\
Hemotórax & $300(61.9 \%)$ & 0.041 \\
Hemoneumotórax & $35(7.21 \%)$ & 1.375 \\
\hline
\end{tabular}

Tabla 3: Análisis multivariado para el desarrollo de empiema como complicación post quirúrgica en trauma de tórax.

\begin{tabular}{lllll}
\hline Variable & $B$ & Sig & OR & $95 \%$ IC \\
\hline Trauma penetrante & 1.632 & 0.10 & 5.080 & $1.48-17.40$ \\
No uso de AB profiláctico & 1.514 & 0.012 & 4.59 & $1.33-15.48$ \\
Hemotorax Coagulado & 3.96 & 0.002 & 52.22 & $16.99-160.5$ \\
\hline
\end{tabular}

continuas. Para la comparación de variables continuas se utilizó el test t-Student, y para las variables categóricas el $\mathrm{Chi}^{2}$ de Pearson, Se consideró significancia estadística una $\mathrm{p} \leq 0.05$.

\section{RESULTADOS}

$\mathrm{Al}$ estudio se incluyeron 485 pacientes con trauma penetrante de tórax el cual fue más frecuente en hombres $411(84.74 \%)$ que en mujeres $74(15.26 \%)$, la media de la edad fue de $34,2 \pm 8,23$ y el mecanismo de trauma más frecuente fue por arma cortopunzante con un $88.1 \%$ seguido por proyectil de arma de fuego con un $10.9 \%$ y empalamiento de $1 \%$. Con respecto al índice de shock se encontró que la media fue de $0,83 \pm 1,02$, el ISS se tuvo una media de $16,4 \pm 2,91$ y el RTS con una media de 9,86 $\pm 2,78$. Las lesiones más frecuentemente encontradas se describen en la tabla número 1.

Tan solo 42 de los pacientes $(8.65 \%)$ desarrollaron algún tipo de complicación postquirúrgica la frecuencia y tipo de complicaciones documentada en la tabla número 2.

Del grupo de pacientes que presentó complicaciones la media de la estancia hospitalaria fue de 14,8 $\pm 2,45$ días. 36 pacientes, es decir, $85.71 \%$ requirieron manejo en la unidad de cuidado intensivo UCI y La media de la estancia UCI fue de $5,38 \pm 1,5$ días, el 66,7\% (28) pacientes requirieron traqueostomía durante la hospitalización en la UCI. El tiempo promedio de ventilación mecánica de este grupo de pacientes fue de 11,3 \pm 1 ,68 días. La mortalidad para el grupo fue del $57.15 \%$ (24). El hemotórax coagulado fue el principal factor de riesgo para el desarrollo de empiema post-traumático (Tabla 3).

De todos los procedimientos quirúrgicos realizados, $318(65.6 \%)$ se llevaron a cabo en el servicio de urgencias y el porcentaje restante 167 (34.4\%) se desarrollaron en quirófanos.

Dentro de los hallazgos de la población general encontramos que el $67,21 \%$ (326) se les realizó toracotomía anterolateral, el 38,1\% (185) se les realizo esternotomía y
Tabla 2: Complicaciones y factores de riesgo relacionadas con el manejo quirúrgico de los pacientes

\begin{tabular}{lll}
\hline & $n=42$ & $P<0.05$ \\
\hline Empiema & $25(59.52 \%)$ & 0.027 \\
Neumonía & $12(28.57 \%)$ & 0.841 \\
SDRA & $5(14.91 \%)$ & 1.295 \\
\hline
\end{tabular}

toracostomia cerrada, el 78,55\% (381) fueron manejados con toracostomía cerrada, el 19,17\% (93) de los pacientes se les realizó esternotomía y toracotomía. Dentro de las lesiones y compromiso a órganos y estructuras anatómicas del tórax encontramos que $71.13 \%$ (345) se afectó el corazón y pulmón, 8\% (39) hubo lesión de vaso, 15,87\% (77) afección única de corazón. El tiempo expuesto a ventilación mecánica de los pacientes de la población en general fue de 9,7 $\pm 3,2$ días, en 10,72\% (52) de los pacientes se les realizó traqueostomía percutánea. La mortalidad total de la población fue de $6.59 \%$ (32), dentro de los pacientes que presentaron empiema y hemotórax coagulado como complicación el 100\% el manejo se hizo por medio de toracoscopia para decorticacion.

\section{DISCUSIÓN}

El Trauma penetrante de tórax es una patología que afecta principalmente a los hombres en edades reproductivas. ${ }^{1,3,13}$ El mecanismo de trauma más frecuente encontrado en este estudio es por arma cortopunzante lo cual se relaciona con lo publicado en la literatura ${ }^{2} \sin$ embargo, hay estudios en los cuales también se reporta que las heridas por proyectil de arma de fuego podrían llegar a ser más frecuentes ${ }^{3}$ esto posiblemente se deba a la facilidad para la adquisición de armas en determinados países y las características de la violencia propia de cada territorio.

Cuando se realiza la clasificación de la severidad mediante escalas para identificar la severidad del trauma, se ha descrito que los pacientes que presentan peor pronóstico durante el curso clínico de manejo de su patología traumática son aquellos que tienen un ISS elevado en el momento del ingreso ${ }^{11}$ en nuestro estudio se encontró una media de ISS de 16,4 $\pm 2,91$ en el grupo de pacientes que presentaron complicaciones posterior al manejo quirúrgico de su patología traumática. Mario Gasparri et $\mathrm{l}^{11}$ describe que en su estudio evidenciaron mejor sobrevivencia con valores menores a 16, lo cual permitiría concluir que el promedio de nuestro grupo de pacientes tuvo heridas de una severidad considerable lo cual los predispone a tener un peor pronóstico a largo plazo.

Adicionalmente encontramos que el manejo quirúrgico que con mayor frecuencia se realiza para el control de las lesiones secundarias al trauma penetrante de tórax es la toracostomía cerrada con un $78.55 \%$ lo cual 
no difiere de lo publicado por Recep Demirhan et al. ${ }^{2}$ Quienes reportan que el $73.6 \%$ de pacientes con la misma patología traumática previamente descrita requirieron el mismo procedimiento mencionado anteriormente.

Con respecto a las complicaciones secundarias a la realización de procedimientos quirúrgicos en los pacientes que presentan trauma penetrante de tórax se describe que la más frecuente es la neumonía ${ }^{14}$ lo cual no se correlaciona con los hallazgos de nuestro estudio en donde la principal complicación fue el empiema. Para lo cual encontramos que el hemotórax coagulado es un factor de riesgo para el desarrollo de ésta.

La mortalidad descrita para el trauma de tórax en general oscila entre $4-20 \%{ }^{4}$ estando el resultado de la nuestra en $6.59 \%$ lo cual se incluye dentro del rango descrito en la literatura; aunque lo alarmante es que este valor ascendió a $57.15 \%$ en el caso de los pacientes que presentaron algún grado de complicación postquirúrgica. Posiblemente se deba a presencia de lesiones adicionales a la torácica o a la severidad del trauma, dificultad en el manejo inicial, puntajes de severidad del trauma con valores altos hecho que no era objeto de evaluación en este estudio por tanto no se consignó, pero sería interesante estudiar este fenómeno mediante nuevos estudios.

Consideramos que las limitaciones de nuestro estudio se basan en que es observacional retrospectivo, lo cual impide en muchas oportunidades realizar una adecuada recolección de datos con todas las variables con las que se espera trabajar, debido a la no disponibilidad de la información en gran parte de los casos. Adicionalmente, se considera pertinente la realización de un estudio analítico prospectivo y por un periodo de tiempo prudencial que permita establecer realmente cuáles son los factores de riesgo específicos para el desarrollo de las complicaciones postquirúrgicas y de esta manera implementar medidas que permitan un control de éstas y por ende disminución en la morbimortalidad de los pacientes con trauma penetrante de tórax.

Hasta la fecha el trauma, en general, sigue siendo un problema de salud pública, cuya principal causa desencadenante es la violencia. Secundario a lo anterior, se deberían implementar mecanismos de contingencia para el control de lo que desencadena las discrepancias y reducir la incidencia de patología traumática en general. Al igual que es necesario, realizar estudios de mayor complejidad y peso estadístico que permitan establecer mecanismos para optimizar el manejo de los pacientes con trauma penetrante de tórax y que de esa manera se reduzca la morbimortalidad que esta entidad genera.

\section{BIBLIOGRAFÍA}

1. Al-Koudmani I, Darwish B, Al-Kateb K, Taifour Y. Chest trauma experience over eleven-year period at al-mouassat university teaching hospital-Damascus: a retrospective review of 888 cases. J Cardiothorac Surg 2012 Apr 19;7:35.

2. Demirhan R, Onan B, Oz K, Halezeroglu S. Comprehensive analysis of 4205 patients with chest trauma: a 10-year experience. Interact Cardiovasc Thorac Surg 2009 Sep;9(3): 450-453.

3. Tariq UM, Faruque A, Ansari H, Ahmad M, Rashid U, Perveen S, Sharif H. Changes in the patterns, presentation and management of penetrating chest trauma patients at a level II trauma centre in southern Pakistan over the last two decades. Interact Cardiovasc Thorac Surg 2011 Jan;12(1): 24-27.

4. AlEassaEM,Al-MarashdaMJ,ElsherifA,EidHOAbu-ZidanFM. Factors affecting mortality of hospitalized chest trauma patients in United Arab Emirates. J Cardiothorac Surg 2013 Mar 30;8:57.

5. Walker WE, Kapelanski DP, Weiland AP, Stewart JD, Duke JH. Patterns of infection and mortality in thoracic trauma. Ann Surg 1985 Jun;201(6):752-756.

6. Kuhajda I, Zarogoulidis K, Kougioumtzi I, Huang H, Li Q, Dryllis G, Kioumis I, Pitsiou G, Machairiotis N, Katsikogiannis N, et al. Penetrating trauma. J Thorac Dis 2014 Oct;6(Supple 4):S461-S465.

7. Huh J, Wall MJ, Estrera AL, Soltero ER, Mattox KL. Surgical management of traumatic pulmonary injury. Am J Surg 2003 Dec;186(6):620-624.

8. Petrone P, Asensio JA. Surgical management of penetrating pulmonary injuries. Scand J Trauma Resusc Emerg Med 2009 Feb 23;17:8.

9. Edwin F, Tettey M, Sereboe L, Aniteye E, Kotei D, Tamatey M, Entsuamensah K, Delia I, Frimpong-Boateng K. Impalement injuries of the chest. Ghana Med J 2009 Jun;43(2):86-89.

10. Huh J, Wall MJ, Estrera AL, Soltero ER, Mattox KL. Surgical management of traumatic pulmonary injury. Am J Surg 2003 Dec;186(6):620-624.

11. Gasparri M, Karmy-Jones R, Kralovich KA, Patton JH, Arbabi S. Pulmonary tractotomy versus lung resection: viable options in penetrating lung injury. J Trauma 2001 Dec;51(6):1092-1095.

12. Kwiatt M, Tarbox A, Seamon MJ, Swaroop M, Cipolla J, Allen C, Hallenbeck S, Davido HT, Lindsey DE, Doraiswamy VA, et al. Thoracostomy tubes: A comprehensive review of complications and related topics. Int J Crit Illn Inj Sci. 2014 Apr; 4(2):143-155.

13. Botache WF, Ordóñez CA, Badiel M, Sanjuan J, Tejada J, Cepeda MC, Pino LF, Parra MW, Scavo DA, Salamea JC. Herida Por Arma de Fuego Como Predictor Independiente de Mortalidad en Trauma de Tórax. Panam J Trauma Critical Care Emerg Surg 2012;1(3):198-203.

14. Martin MJ, McDonald JM, Mullenix PS, Steele SR, Demetriades D. Operative management and outcomes of traumatic lung resection. J Am Coll Surg 2006 Sep;203(3):336-344. 